\title{
Search for the critical point of strongly interacting matter through power-law fluctuations of the proton density in NA61/SHINE
}

\author{
Nikolaos Davis* for the NA61/SHINE Collaboration \\ Institute of Nuclear Physics, Polish Academy of Sciences \\ E-mail: nikolaos.daviseifj.edu.pl
}

\section{Nikolaos Antoniou}

University of Athens, Physics Department, Section of Nuclear and Elementary Particle Physics

E-mail: nantonio@phys.uoa.gr

\section{Fotios Diakonos}

University of Athens, Physics Department, Section of Nuclear and Elementary Particle Physics

E-mail: fdiakono@phys.uoa.gr

The search for experimental signatures of the critical point (CP) of strongly interacting matter [1] is one of the main objectives of the NA61/SHINE experiment at the CERN SPS. In the course of the experiment, an energy (beam momentum $13 \mathrm{~A}-150 / 158 \mathrm{~A} \mathrm{GeV} / \mathrm{c}$ ) and system size $(\mathrm{p}+\mathrm{p}, \mathrm{p}+\mathrm{Pb}, \mathrm{Be}+\mathrm{Be}, \mathrm{Ar}+\mathrm{Sc}, \mathrm{Xe}+\mathrm{La})$ scan is performed. Proposed observables include nonmonotonic fluctuations of integrated quantities, as well as local critical fluctuations connected to the critical behavior of the order parameter in the neighborhood of the $\mathrm{CP}$, which scale according to universal power-laws.

We investigate proton density fluctuations as a possible order parameter of the phase transition in the neighborhood of the CP. To this end, we perform an intermittency analysis of the proton second scaled factorial moments (SSFMs) in transverse momentum space. A previous analysis of this sort [2] revealed significant power-law fluctuations in the NA49 heavy ion collision experiment for the "Si" $+\mathrm{Si}$ system at $158 \mathrm{~A} \mathrm{GeV/c}$. The fitted power-law exponent was consistent with the theoretically expected critical value, within errors, a result suggesting a baryochemical potential for the critical point in the vicinity of $250 \mathrm{MeV}$. We now extend the analysis to NA61 systems of similar size, $\mathrm{Be}+\mathrm{Be}$ and $\mathrm{Ar}+\mathrm{Sc}$, at $150 \mathrm{~A} \mathrm{GeV/c}$.

We adapt statistical techniques for the calculation of scaled factorial moments, in order to subtract non-critical background and enhance the signal in cases of low statistics. Our analysis is supplemented by both critical and non-critical Monte Carlo simulations, through which we estimate non-critical background effects on the quality and magnitude of uncertainties of the intermittency power-law fit, as well as explore the possibility of non-critical effects producing an intermittency signal.

PACS: $24.10 . \mathrm{Lx}, 25.75 .-q, 25.75 . \mathrm{Gz}, 25.75 . \mathrm{Nq}$

Critical Point and Onset of Deconfinement - CPOD2017

7-11 August, 2017

The Wang Center, Stony Brook University, Stony Brook, NY

${ }^{*}$ Speaker. 


\section{Introduction}

Experimental observables proposed for the detection of the QCD CP fall into two categories: event-by-event (global) fluctuations of integrated quantities [3, 4, 5], as well as local power-law fluctuations [6] of the order parameter of the QCD chiral phase transition, the chiral condensate $\langle\bar{q} q\rangle$. The critical properties of the chiral condensate are carried by the sigma field $\sigma(\mathbf{x})$, and may be probed indirectly through its decay into experimentally observable $\left(\pi^{+}, \pi^{-}\right)$pairs [7]. At finite baryochemical potential, critical fluctuations are also transferred to the net proton density, $n_{B}(\mathbf{x})$, as well as to the proton and antiproton densities separately [8].

At the CP, the fluctuations of the order parameter are self-similar [9], belonging to the 3D-Ising universality class. Through a Fourier transform, long-range correlations of the order parameter in transverse configuration space correspond to power-law correlations in transverse momentum space in the limit of small momentum transfer, $\mathbf{k} \rightarrow 0$. The latter can be detected within the framework of an intermittency analysis of proton density fluctuations in transverse momentum space, by use of scaled factorial moments (SFMs). A detailed analysis, augmented by properly adapted statistical techniques, can be found in [2], where we study various heavy nuclei collision datasets recorded in the NA49 experiment at maximum energy (158A GeV, $\sqrt{s_{N N}} \approx 17 \mathrm{GeV}$ ) of the SPS (CERN).

\section{Method of analysis}

In a pure critical system, intermittency in transverse momentum space can be revealed by the scaling of the Second Scaled Factorial Moments (SSFMs) of protons as a function of bin size. For that purpose, a region of transverse momentum space is partitioned into $M \times M$ equal-size bins. Consequently, the SSFMs:

$$
F_{2}(M)=\frac{\left\langle\frac{1}{M^{2}} \sum_{i=1}^{M^{2}} n_{i}\left(n_{i}-1\right)\right\rangle}{\left\langle\frac{1}{M^{2}} \sum_{i=1}^{M^{2}} n_{i}\right\rangle^{2}}
$$

are calculated, as an average over bins and events $(\langle\ldots\rangle)$, where $n_{i}$ is the number of particles in the $i$-th bin and $M^{2}$ is the total number of bins. If the system exhibits critical fluctuations, $F_{2}(M)$ is expected to scale with $M$, for large values of $M$, as a power-law:

$$
F_{2}(M) \sim M^{2 \phi_{2}}, \phi_{2}=\phi_{2, c r}^{B}=\frac{5}{6}
$$

where $\phi_{2}$ is the intermittency index, and provided the freeze-out occurs at exactly the critical point [10].

Noisy experimental data require the subtraction of a background of uncorrelated \& misidentified protons, which is achieved through the construction of correlation-free mixed events. A correlator can then be defined in terms of the moments of data and mixed events, as well as a mixed term, 


$$
\Delta F_{2}(M)=F_{2}^{(d)}(M)-\lambda^{2}(M) F_{2}^{(m)}(M)-\lambda(M)(1-\lambda(M)) f_{b c} \quad ; \quad \lambda(M) \equiv \frac{\left\langle n_{b}\right\rangle}{\langle n\rangle}
$$

where $\lambda(M)$ is the ratio of background to data average particle multiplicities and $f_{b c}$ an unknown function. In the limiting case of a dominant background, $\lambda(M) \lesssim 1$, the critical behaviour is expected to be revealed in the approximate ${ }^{1}$ correlator,

$$
\Delta F_{2}^{(e)}(M) \simeq F_{2}^{(d)}(M)-F_{2}^{(m)}(M),
$$

where mixed event $(m)$ moments are simply subtracted from data $(d)$ moments. $\Delta F_{2}(M)$ should then scale as a power law, $\Delta F_{2}(M) \sim M^{2 \phi_{2}}$, in a limited range, with the same intermittency index as the pure critical system.

Furthermore, calculation of SSFMs is smoothed by averaging over many lattice positions (lattice averaged SSFMs, see [2]). An improved estimation of statistical errors of SSFMs is achieved by use of the bootstrap method $[11,12,13]$, whereby the original set of events is resampled with replacement [2].

A proton generating modification of the Critical Monte Carlo (CMC) code $[6,10]$ is used to simulate a system of critically correlated protons, which are mixed with a non-critical background to study the effects on the quality of intermittency analysis.

\section{Results}

\subsection{NA49 data analysis results}

An intermittency analysis performed on a number of different size NA49 collision systems at the maximum SPS energy [2] at mid-rapidity detected power-law fluctuations compatible with criticality in the "Si" $+\mathrm{Si}$ system, although with large statistical errors. Furthermore, no intermittency was detected in the " $\mathrm{C}$ " $+\mathrm{C}$ and $\mathrm{Pb}+\mathrm{Pb}$ systems at the same collision energy.

An interesting result emerges from simulating the NA49 "Si"+Si system, by mixing random (uncorrelated) simulated protons with critical protons produced via the aforementioned CMC code. The average ratio $\lambda$ of random noise to total proton event multiplicity is an adjustable parameter of such a simulation. Fig.1(a) shows the moments $F_{2}(M)$ of NA49 "Si" $+\mathrm{Si}$ data compared to a $\lambda=99 \%$ noise-contaminated CMC set, which approximates the former with fair accuracy. In Fig.1(b), $\Delta F_{2}^{(e)}(M)$ is shown for the same CMC set, as well as the pure CMC; we notice that $\Delta F_{2}^{(e)}(M)$ of noisy CMC reproduces the slope (intermittency index $\phi_{2}$ ) of the pure CMC critical set, although their moments differ by orders of magnitude. Finally, Fig.1(c) shows the distribution of $\phi_{2}$ values across different bootstrap samples for the same noisy CMC set. The spread of $\phi_{2}$ values in the NA49 "Si"+Si system is reproduced and justified through mixing with background protons, whereas the median $\phi_{2}$ value approximates the theoretically expected intermittency index of the pure CMC. We conclude from noisy CMC simulations that indeed background is dominant in the NA49 data sets, and that the $\Delta F_{2}^{(e)}(M)$ approximation is valid in the case of dominant background.

\footnotetext{
${ }^{1}$ This approximation is non-trivial to derive; the mixed term can be shown to be negligible through use of CMC simulation mixed with non-critical background.
} 

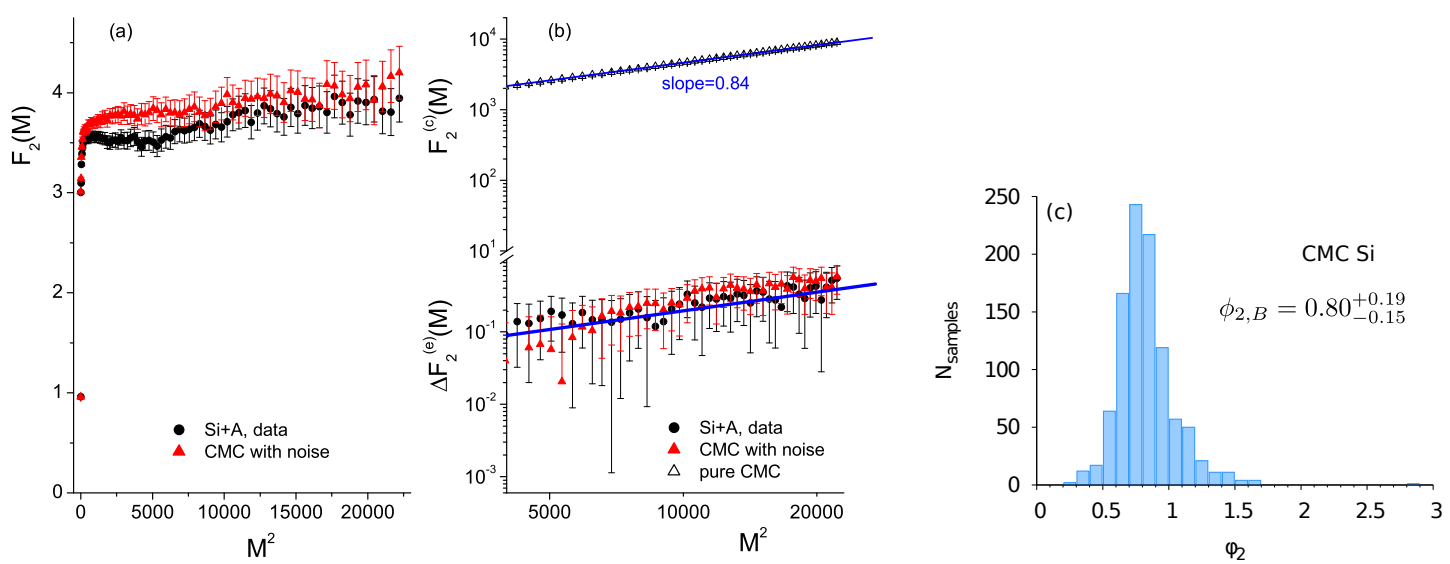

Figure 1: (a) $F_{2}(M)$ of NA49 "Si" $+\mathrm{Si}$ data (filled cirles) and noisy CMC simulated collisions (filled triangles) with $\lambda=99 \%$ background noise. (b) $F_{2}^{(c)}(M)$ for pure CMC system (open triangles), as well as the correlator $\Delta F_{2}^{(e)}(M)$ for noisy CMC. $\Delta F_{2}^{(e)}(M)$ is also shown for NA49 "Si"+Si data for comparison. The solid lines correspond to the theoretically expected slope $\phi_{2, c r}^{B}$. (c) $\phi_{2, B}$ bootstrap distribution for noisy "Si"+Si CMC. Asymmetric errors correspond to a $67 \%$ confidence interval.

\subsection{NA61/SHINE Be+Be preliminary data analysis}

Following the positive NA49 "Si"+Si result, we are encouraged to look for intermittency in collisions of medium-sized nuclei recorded within the successor experiment NA61/SHINE. In order to reliably perform an intermittency analysis, we require large event statistics (a minimum of $100 \mathrm{~K}$ events, ideally of the order of $1 \mathrm{M}$ events), reliable particle identification (candidate proton purity of at least $80-90 \%$ ), and a relatively high mean proton multiplicity density in mid-rapidity for $5-10 \%$ most central collisions (typically, $d N_{p} / d y \geq 1.5 \rightarrow 2$ for $\left|y_{C M}\right| \leq 0.75, p_{T} \leq 1.5 \mathrm{GeV} / \mathrm{c}$ ). Subsequently, our two main candidate NA61 systems for study are ${ }^{7} \mathrm{Be}+{ }^{9} \mathrm{Be}$ and ${ }^{40} \mathrm{Ar}+{ }^{45} \mathrm{Sc}$ at $150 \mathrm{~A} \mathrm{GeV/c}$.

Preliminary analysis of NA61 $\mathrm{Be}+\mathrm{Be}$ data reveals that

$$
\left.\frac{d N_{p}}{d y}\right|_{\left|y_{C M}\right| \leq 0.75, p_{T} \leq 1.5 \mathrm{GeV} / \mathrm{c}} \sim 0.7
$$

a rather low value for the standards of an intermittency analysis. We therefore do not expect to observe an intermittency effect in $\mathrm{Be}+\mathrm{Be}$, but we will attempt to establish an upper limit for the critical component fraction in $\mathrm{Be}+\mathrm{Be}$.

We analyzed a set of about $160 \mathrm{~K}, 10 \%$ most central NA61/SHINE Be+Be collisions at $150 \mathrm{GeV} / \mathrm{c}$ beam momentum per nucleon, corresponding to a CM energy of $\sqrt{s_{N N}}=16.8 \mathrm{GeV}$. The average proton multiplicity per event was $1.48 \pm 0.74$ in the mid-rapidity range, excluding events with a zero proton multiplicity in this range. Based on these results, we also simulated a similar number of noisy CMC events, for various noise levels $\lambda$, and with a Poisson-distributed proton multiplicity with $\mu_{\text {Poisson }}=0.76$, which reproduces the $\mathrm{Be}+\mathrm{Be}$ multiplicity distribution 
for the set of non-empty events. The one-particle transverse momentum distribution of $\mathrm{Be}+\mathrm{Be}$ is also plugged in as input parameter to the CMC simulation.

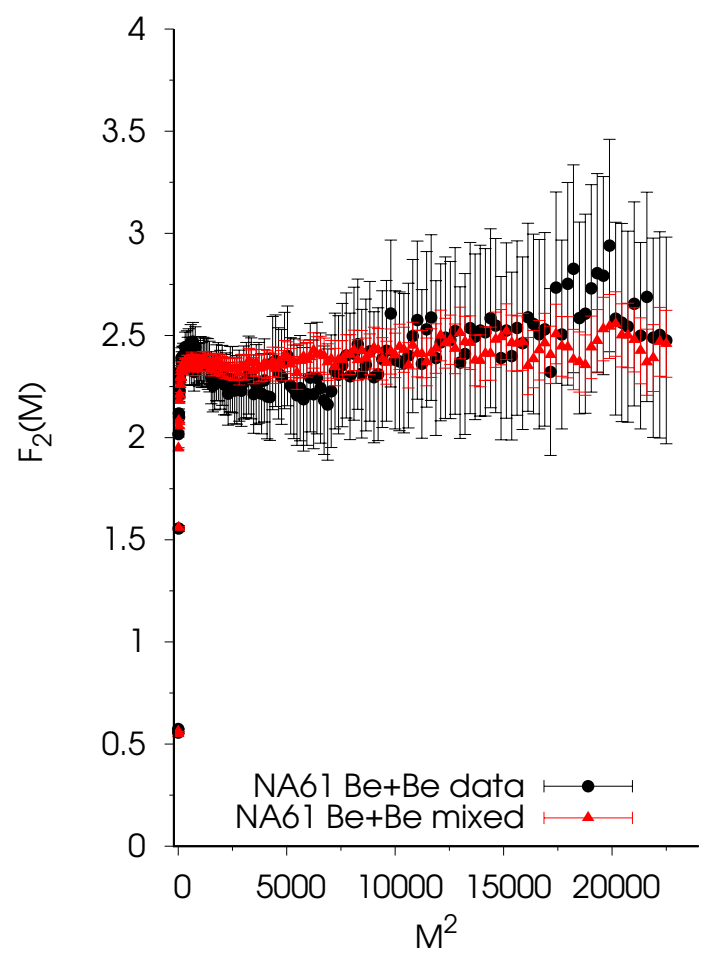

Figure 2: $F_{2}(M)$ of protons in NA61/SHINE Be+Be collisions at $\sqrt{s_{N N}}=16.8 \mathrm{GeV}$, for data (black circles) and mixed events (red triangles).

In Fig.2, intermittency analysis results are shown for NA61/SHINE Be+Be data. $F_{2}(M)$ for data and mixed events overlap; thus, $\Delta F_{2}(M)$ fluctuates around zero, and no intermittency effect is observed. Fig. 3 shows the results of the CMC for several levels of noise. We see that $F_{2}(M)$ of noisy $\mathrm{CMC}$ approximates $\mathrm{Be}+\mathrm{Be}$ data for a $99.7 \%$ noise level, for which, however, the corresponding $\Delta F_{2}(M)$ has deteriorated far too much to allow us to obtain an intermittency index. Based on this result, we estimate an upper limit of the order of $0.3 \%$ for the fraction of critical protons in $\mathrm{Be}+\mathrm{Be}$ data. We note that intermittency analysis is sensitive enough to detect a tiny subset of the data that exhibits critical scaling, provided that enough statistics are used.

\subsection{Feasibility study for Ar+Sc in NA61/SHINE}

$\mathrm{Ar}+\mathrm{Sc}$ analysis of NA61 data is still in progress; however, simulations performed with the EPOS event generator [14], for a maximum impact parameter $b \sim 3.5 \mathrm{fm}$, suggest

$$
\left.\frac{d N_{p}}{d y}\right|_{\left|y_{C M}\right| \leq 0.75, p_{T} \leq 1.5 \mathrm{GeV} / c} \sim 4,
$$

quite adequate for an intermittency analysis.

We simulated in EPOS a set of $100 \mathrm{~K}$ Ar+Sc collision events at $150 \mathrm{GeV} / \mathrm{c}$ beam momentum per nucleon, corresponding to a $\mathrm{CM}$ energy of $\sqrt{s_{N N}}=16.8 \mathrm{GeV}$. The average proton multiplicity 

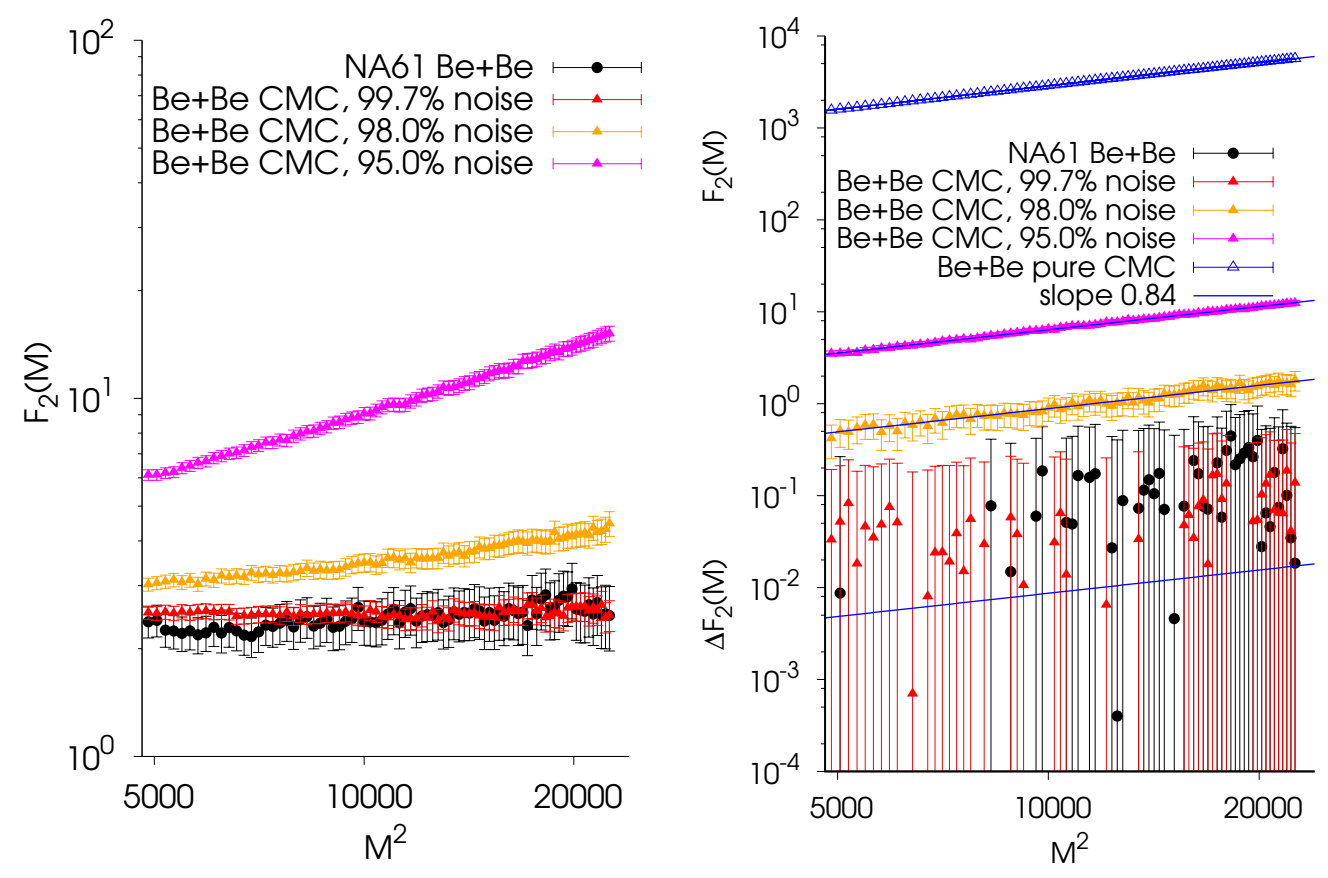

Figure 3: SSFMs $F_{2}(M)$ (Left) and $\Delta F_{2}(M)$ (Right) of NA61/SHINE Be+Be collisions (10\% most central, $\left.\sqrt{s_{N N}}=16.8 \mathrm{GeV}\right) \&$ noisy CMC Be+Be simulated collisions. For comparison, $F_{2}(M)$ of pure CMC is plotted, along with the theoretically expected slope.

per event was $5.3 \pm 2.5$ in the mid-rapidity range, excluding events with a zero proton multiplicity in this range. As in $\mathrm{Be}+\mathrm{Be}$, we simulated a similar number of noisy $\mathrm{CMC}$ events, for various noise levels $\lambda$, with $\mu_{\text {Poisson }}=5.3$, matching the EPOS proton multiplicity distribution.

Analysis results are shown in Fig.4. We see that $F_{2}(M)$ of noisy CMC approximates EPOS $\mathrm{Ar}+\mathrm{Sc}$ for a $99.5 \%$ noise level; however, in this case the corresponding $\Delta F_{2}(M)$ retains a fair power-law quality that allows $\phi_{2}$ to be recovered. Fitting the correlator $\Delta F_{2}(M)$ for CMC for all bootstrap samples, we obtain a confidence interval for $\phi_{2}$ that is consistent with the plug-in theoretically expected value of 5/6. Therefore, CMC combined with EPOS simulation indicates that an intermittency signal would become visible in $\mathrm{Ar}+\mathrm{Sc}$ for a critical proton fraction of about $0.5 \%$.

\section{Summary and conclusions}

The study of self-similar (power-law) fluctuations of the proton density in transverse momentum space through intermittency analysis provides us with a promising set of observables for the detection of the QCD critical point. The intermittency analysis performed on NA49 "Si"+Si data at the maximum SPS energy already indicates the presence of a critical proton component of the order of $1 \%$, with an estimated intermittency index value of $\phi_{2, B}=0.96_{-0.25}^{+0.38}$, overlapping with the critical QCD prediction, whereas no intermittency is observed in either the smaller " $\mathrm{C}$ " $+\mathrm{C}$ or the larger $\mathrm{Pb}+\mathrm{Pb}$ system at the same collision energy. 

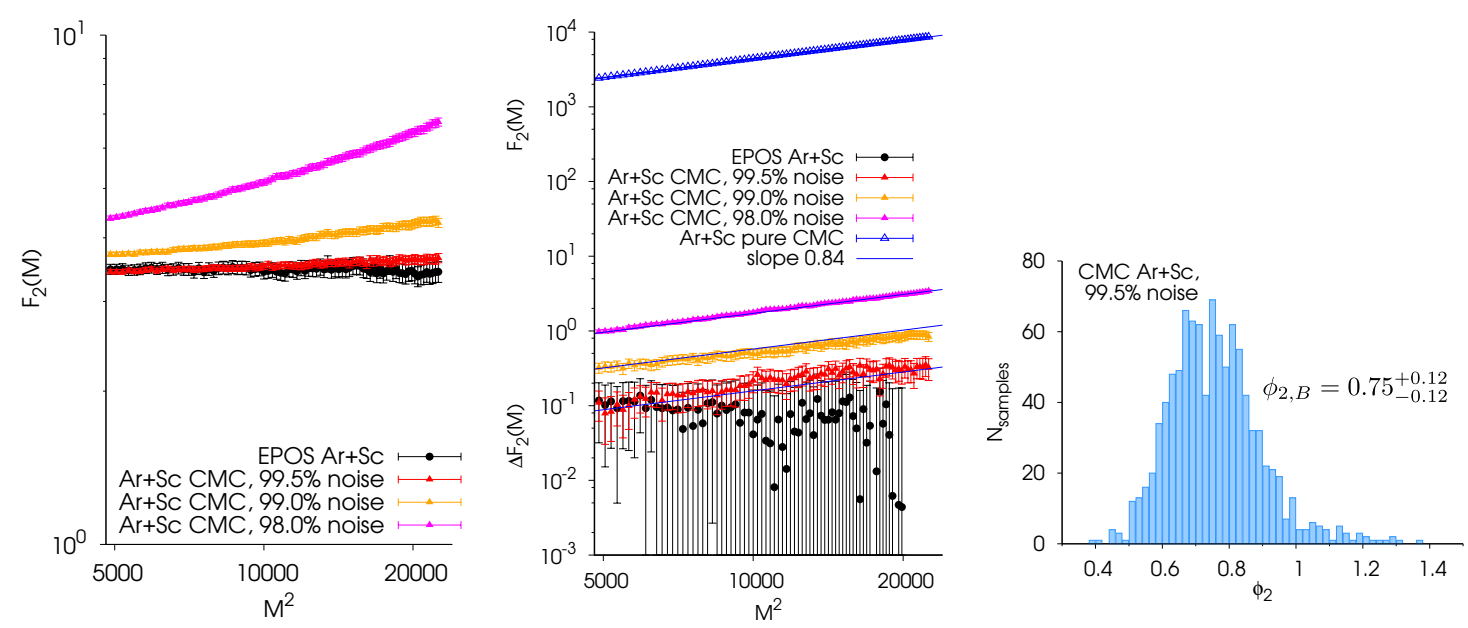

Figure 4: SSFMs $F_{2}(M)$ (Left) and $\Delta F_{2}(M)$ (Middle) of EPOS \& noisy CMC Ar+Sc simulated collisions ( $10 \%$ most central, $\left.\sqrt{s_{N N}}=16.8 \mathrm{GeV}\right)$. For comparison, $F_{2}(M)$ of pure CMC is plotted, along with the theoretically expected slope. Right: Distribution of $\phi_{2}$ values, obtained via bootstrap resampling of events.

A preliminary analysis of NA61 $\mathrm{Be}+\mathrm{Be}$ central collisions at $150 \mathrm{GeV} / \mathrm{c}$ beam momentum per nucleon indicates an upper limit of about $0.3 \%$ critical protons; feasibility studies in $\mathrm{Ar}+\mathrm{Sc}$ collisions at the same beam momentum indicates a sensitivity level to a critical component of about $0.5 \%$. Based on these results, we estimate an intermittency analysis to be feasible for (at least) the Ar+Sc system at maximum SPS energy. Systematically extending this analysis to other system sizes (Xe+La) and energies of the NA61/SHINE program will hopefully lead to an accurate determination of the critical point location.

Acknowledgments: This work was supported by the National Science Centre, Poland (grant no. 2014/14/E/ST2/00018).

\section{References}

[1] K. Fukushima and T. Hatsuda, Rep. Prog. Phys. 74, 014001 (2011).

[2] T. Anticic et al, Eur. Phys. J. C 75: 587 (2015).

[3] C. Alt et al., Phys. Rev. C 75, 064904 (2007); Phys. Rev. C 78, 034914 (2008).

[4] L. Adamczyk et al., Phys. Rev. Lett. 112, 032302 (2014).

[5] T. Anticic et al., Phys. Rev. C 70, 034902 (2004); Phys. Rev. C 79, 044904 (2009).

[6] N. G. Antoniou, Y. F. Contoyiannis, F. K. Diakonos, A. I. Karanikas and C. N. Ktorides, Nucl. Phys. A 693, 799 (2001).

[7] N. G. Antoniou, Y. F. Contoyiannis, F. K. Diakonos and G. Mavromanolakis, Nucl. Phys. A 761, 149 (2005).

[8] Y. Hatta and M. A. Stephanov, Phys. Rev. Lett. 91, 102003 (2003).

[9] T. Vicsek, Fractal Growth Phenomena (World Scientific, Singapore, 1989). ISBN 9971-50-830-3 
[10] N. G. Antoniou, F. K. Diakonos, A. S. Kapoyannis and K. S. Kousouris, Phys. Rev. Lett. 97, (2006) 032002 .

[11] W. J. Metzger, "Estimating the Uncertainties of Factorial Moments", HEN-455 (2004).

[12] B. Efron, Ann. Stat. 7, 1 (1979).

[13] T. Hesterberg et al., Bootstrap Method and Permutation Tests (W. H. Freeman \& Co., USA, 2003). ISBN-10:0716757265

[14] K. Werner, F. Liu, T. Pierog, Phys. Rev. C 74, 044902 (2006). 